\title{
INEQUALITIES FOR ALMOST PERIODIC MEASURES
}

\author{
SilviA-OTILIA CORDUNEANU
}

Abstract. Some inequalities for the mean of certain almost periodic measures are presented. We give inequalities like Hölder, Minkowski or Jensen, for the mean of almost periodic measures defined by densities.

Mathematics subject classification (2000): 43A60, $26 \mathrm{D} 15$.

Key words and phrases: Almost periodic measure, almost periodic function, mean, inequality.

\section{REFERENCES}

[1] L. N. ARgabright AND J. GiL DE LAmAdRID, Fourier Analysis of Unbounded Measures on Locally Compact Abelian Groups, Mem. Amer. Math. Soc. No. 145 (1974).

[2] L. N. Argabright And J. Gil de Lamadrid, Almost Periodic Measures, Mem. Amer. Math. Soc. No. 428 (1990).

[3] E. Beckenbach, R. Bellman, Inequalities, Springer-Verlag, Berlin, Heidelberg, New York, Tokyo, 1983.

[4] N. Dinculeanu, Vector Measures, Veb Deutscher Verlag Der Wissenschaften, Berlin, 1966.

[5] N. Dinculeanu, Integrarea pe Spaţii Local Compacte, Editura Academiei R. P. R., Bucureşti, 1965 (Romanian).

[6] G. H. Hardy, J. E. Littlewood, G. Pólya, Inequalities, Cambridge Univ. Press, 1934.

[7] E. Hewitt AND K. A. Ross, Abstract Harmonic Analysis Vol. I, Springer-Verlag, Berlin, Göttingen, Heidelberg, 1963.

[8] J. GIL DE LAMADRID, Sur les Mesures Presque Périodiques, Séminaire KGB sur les marches aléatoires, Astérisque 4, 1973.

[9] D. S. Mitrinović, Analytic Inequalities, Springer-Verlag, Berlin, Heidelberg, New York, 1970.

[10] W. Rudin, Fourier Analysis on Groups, Interscience Tracts in Pure and Applied Mathematics, Number 12, Interscience Publishers - John Wiley and Sons, New York, London, 1962.

[11] A. Zygmund, Trigonometric Series, Vol. I, Cambridge, 1959. 